\title{
Recommendations and Practical Guidance for Performing and Reporting Validation Studies according to the Universal Standard for the Validation of Blood Pressure Measuring Devices by the Association for the Advancement of Medical Instrumentation/European Society of Hypertension/International Organization for Standardization (AAMI/ESH/ISO)
}

Short title: Blood pressure monitors validation

\author{
George Stergiou ${ }^{1}$, Paolo Palatini ${ }^{2}$, Roland Asmar ${ }^{3}$, John P. Ioannidis ${ }^{4}$, Anastasios Kollias ${ }^{1}$, \\ Peter Lacy ${ }^{5}$, Richard J McManus ${ }^{6}$, Martin Myers ${ }^{7}$, Gianfranco Parati ${ }^{8}$, Andrew Shennan ${ }^{9}$, \\ Jiguang Wang ${ }^{10}$, Eoin $\mathrm{O}^{\prime}$ Brien $^{11}$, on behalf of the \\ European Society of Hypertension Working Group on Blood Pressure Monitoring
}

\footnotetext{
${ }^{1}$ Hypertension Center STRIDE-7, National and Kapodistrian University of Athens, School of Medicine, Third Department of Medicine, Sotiria Hospital, Athens, Greece.

${ }^{2}$ Department of Medicine. University of Padova, Italy.

${ }^{3}$ Foundation, Medical Research Institutes, Paris France.

${ }^{4}$ Departments of Medicine, of Health Research and Policy, and of Biomedical Data Science, Stanford University School of Medicine, and Department of Statistics, Stanford University School of Humanities and Sciences, Stanford, USA.

${ }^{5}$ Institute of Cardiovascular Science, University College London and the National Institute for Health Research University College London Hospitals Biomedical Research Centre, UK.

${ }^{6}$ Green Templeton College, Nuffield Department of Primary Care Health Sciences, University of Oxford, Oxford, UK.

${ }^{7}$ University of Toronto, Schulich Heart Program. Division of Cardiology, Sunnybrook Health Sciences Centre, Toronto, Canada.

${ }^{8}$ Department of Medicine and Surgery, University of Milano-Bicocca; Istituto Auxologico Italiano, IRCCS, Cardiology Unit and Department of Cardiovascular, Neural and Metabolic Sciences, S.Luca Hospital, Milano, Italy.

${ }^{9}$ Department of Women and Children's Health, School of Life Course Sciences, FoLSM, Kings College, London, UK.

${ }^{10}$ Shanghai Institute of Hypertension, Department of Hypertension, Centre for Epidemiological Studies and Clinical Trials, Ruijin Hospital, Shanghai Jiaotong University School of Medicine, Shanghai, China.

${ }^{11}$ The Conway Institute, University College Dublin, Ireland.
}

Source of funding: none

Conflict of interest: GS, PP, RA, GP, EOB: conducted validation studies for various manufacturers; advised manufacturers on device and software development. RM: Received blood pressure monitoring equipment for research purposes from Omron and Lloyds Pharmacies; Chair of British Hypertension Society Blood Pressure Monitoring Working Party which oversees validation studies for various manufacturers. AS: Conducted validation studies for various manufacturers; developed the CRADLE VSA. JW: Conducted validation studies for various manufacturers. JI, AK, PL, MM: none. 
Word count: Abstract: $156 \quad$ Manuscript: 6,020 (with references and tables)

Tables: 13

Figures: 3

Correspondence: Prof. George S. Stergiou, Hypertension Center STRIDE-7,

National and Kapodistrian University of Athens, School of Medicine, Third Department of Medicine, Sotiria Hospital.

152 Mesogion Avenue, Athens 11527, Greece.

Tel: +30 2107763117, Fax: +30 2107719981

Email: gstergi@med.uoa.gr

\begin{abstract}
In the last 30 years several organizations have developed protocols for clinical validation of blood pressure measuring devices. An international initiative was recently launched by the US Association for the Advancement of Medical Instrumentation (AAMI), the European Society of Hypertension Working Group on Blood Pressure Monitoring (ESH) and the International Organization for Standardization (ISO), aiming to reach consensus on a universal AAMI/ESH/ISO validation standard. The purpose of this statement by the ESH Working Group on Blood Pressure Monitoring is to provide practical guidance for investigators performing validation studies according to the AAMI/ESH/ISO Universal Standard (ISO 81060-2:2018), to ensure that its stipulations are meticulously implemented and data are fully reported. Thus, this statement provides: (i) a list of key recommendations for validation studies of intermittent non-invasive automated blood pressure measuring devices according to the AAMI/ESH/ISO Universal Standard, (ii) practical stepwise guidance for researchers performing these validation studies, (iii) a checklist for authors and reviewers of such studies.
\end{abstract}

Keywords: accuracy, blood pressure measurement, device, monitor, protocol, standard, validation 


\section{Background}

Documenting the accuracy of blood pressure (BP) measuring devices is fundamental for the reliable diagnosis and efficient management of hypertension and other medical conditions. In the last 30 years, several organizations such as the US Association for the Advancement of Medical Instrumentation (AAMI), the British Hypertension Society, the European Society of Hypertension (ESH) Working Group on BP Monitoring, the German Hypertension League and the International Organization for Standardization (ISO), have put considerable effort into establishing minimum standards of accuracy and performance for BP monitors [1,2].

In 2016, an international initiative was launched by the AAMI, the ESH and the ISO, with the purpose of drawing on past experience to achieve consensus on a universal validation procedure [3]. In 2018, an AAMI/ESH/ISO statement of intention was published, presenting the global principles and key elements of such a protocol, and stipulating that it would replace all previous ones [3]. Since then, the AAMI/ESH/ISO Universal Standard (ISO 81060-2:2018) for the validation of BP measuring devices has been completed and is available at the ISO [4] and the AAMI websites [5]. The AAMI, ESH and ISO membership comprises medical doctors with expertise in BP monitoring research, methodologists, and technical staff from medical device manufacturers, all of whom worked together over many months to reach consensus on this Universal Standard for the validation of BP measuring devices [3]. To allow for completion and publication of ongoing validation studies using previous protocols, the AAMI/ESH/ISO Universal Standard will be implemented as the mandatory international standard for BP measuring devices one year after the date of its publication.

The ESH Working Group on BP Monitoring has a long history in developing validation procedures for worldwide use and in providing guidance to investigators for the accurate implementation and complete reporting of BP monitor validation studies [6-8]. With the establishment and publication of the Universal Standard, it is important to provide practical guidance for investigators performing validation studies to ensure that its stipulations are meticulously implemented and data are fully reported.

The purpose of this statement from the ESH Working Group on BP Monitoring is to deliver: (i) a list of all the key recommendations for validation studies of intermittent non-invasive automated BP measuring devices according to the AAMI/ESH/ISO Universal Standard (ISO 810602:2018),

(ii) a practical stepwise guidance for researchers performing such validation studies and (iii) a checklist for authors and reviewers of these validation studies to ensure complete data reporting according to the AAMI/ESH/ISO Universal Standard.

It is not the purpose of this statement to discuss the rationale and supporting evidence for the requirements included in the AAMI/ESH/ISO Universal Standard (ISO 81060-2:2018), nor to present information on the mathematical and statistical background and formulae used for its calculations; these details are available in the ISO and AAMI publications [4,5]. Likewise, considerations of the opposite arm simultaneous method of validation, the use of reference invasive BP, the additional requirements for devices intended for ambulatory monitoring or exercise stress testing and validations in children aged $<3$ years are not discussed in this paper but are included in the ISO and AAMI publications $[4,5]$. This standard is not appropriate for validating continuous cuffless BP monitors or central BP monitors for which other validation procedures will be developed [3].

Validation study registration 
- Transparency in the process of evaluating the accuracy of BP monitors is essential. Prospective registration of validation studies should become mandatory for devices that have been brought to the market to ensure that those that failed validation are not recommended and withdrawn, even when the validation results are not published. The investigators and manufacturers should be encouraged to share the raw data of validation studies.

\section{Validation team}

- Observers: Two (or more) health-care professionals trained in auscultatory BP measurement will be qualified for their accuracy against an expert observer and against each other immediately before the validation study initiation using the following procedure: $10 \mathrm{BP}$ measurements are made by the observers simultaneously and blinded to each-other's readings (two Y-tube connected auscultatory devices with single inflation system [see below 'Reference BP measurement - Devices']; dual-head stethoscope; deflation rate 2-3 $\mathrm{mmHg} / \mathrm{s}$; Korotkoff $\mathrm{K} 1$ for systolic [SBP] and K5 for diastolic BP [DBP]) on each of 5 subjects (total of 50 measurements) with a BP range from $110 / 60$ to $190 / 110 \mathrm{mmHg}$ [9]. Forty-five of the resulting $50 \mathrm{SBP}$ and DBP differences between observers must be $\leq 5 \mathrm{mmHg}$ and 48 differences $\leq 10 \mathrm{mmHg}$ [9]. Failure to achieve this level of agreement necessitates repeat training of the observers and re-assessment. If disagreement persists audiograms will be performed.

- Supervisor: An individual experienced in validation protocols must supervise the entire validation procedure, including assessing the adequacy of reference and test device BP measurements and taking the BP measurements with the test device.

\section{Validation procedure}

- Conditions: The validation study must be performed in an isolated and quiet room at a comfortable temperature for the individual participant. The validation procedure starts with the subject seated comfortably and relaxed for at least $5 \mathrm{~min}$, her/his back and arm supported with the middle of the upper arm at heart level, legs uncrossed and feet flat on the floor. The subject will be asked to empty his/her bladder prior to the validation session. Talking and any other interference must be avoided throughout the entire validation procedure.

- Procedure: The same arm sequential BP measurement method is the recommended standard for validation (Table 1) [2-6,9]. With the same arm sequential method either arm may be used.

- The opposite arm simultaneous method can also be used as presented in the ISO 81060-2:2018 $[4,5]$. One of the 2 observers' records the subject's heart rate (also checks for potential arrhythmia) and a Reference BP measurement $(R O)$ is taken simultaneously by the 2 observers (see below 'Reference BP measurement'). Then the cuff is changed and a Test device measurement $(T O)$ is taken (Table 1). Then 4 Reference BP measurements follow (R1-R4), alternated by 3 Test device measurements (R1-T1-R2-T2-R3-T3-R4). Measurements are performed with 60-s intervals from the change of the cuff to the start of the next reading.

\section{Reference BP measurement}

- Method: The auscultatory method is used for reference BP with measurements taken simultaneously by 2 trained observers (two Y-tube connected devices with single inflation system; cuff on bare arm; dual-head stethoscope) blinded to the readings of each-other and of the test device. The average of the 2 observers' measurements is the reference BP. Korotkoff K1 is used for reference SBP and K5 for DBP (deflation rate $2-3 \mathrm{mmHg} / \mathrm{s}$ ) in all subjects (adults, children aged $\geq 3$ years, pregnancy). If $\mathrm{K} 1$ or $\mathrm{K} 5$ is not audible, the subject is excluded. Parallax errors, terminal digit preference and rounding must be avoided. 
- Devices: Mercury sphygmomanometers must be calibrated at the beginning of each validation study. Nonmercury auscultatory manometers (aneroid or other) that fulfill the ISO 81060-1 requirements and with maximum permissible error $\pm 1 \mathrm{mmHg}$ can also be used [3-5]. The accuracy of non-mercury devices must be evaluated against a mercury sphygmomanometer or a calibrated and certified pressure device at the beginning of each validation study. National metrology institutes and other institutions might provide such calibration services. The reference device type, manufacturer and calibration must be reported.

- Cuffs: The cuffs used for reference auscultatory BP measurement must have an inflatable bladder length that covers $75-100 \%$ of the upper arm circumference of each subject and width that covers $37-50 \%$ of the arm circumference [3]. The arm circumference must be measured at the upper arm midpoint (marking the arm posteriorly at a point halfway between the highest [acromion] and lowest bony point [olecranon]) while the arm is hanging freely by the side. An example of cuff use (inflatable bladder dimensions) for reference auscultatory BP measurement in a general population validation study including children is shown in Table 2. The test device cuffs must not be used for reference BP measurements. The inflatable bladder dimensions of the cuffs used for reference BP measurement must be reported.

- Observer agreement: The supervisor will review each pair of Reference/Test BP measurements. If any of them needs to be excluded (due to observer disagreement $>4 \mathrm{mmHg}$ in simultaneous SBP or DBP measurement, or due to test device failure), then the exclusions must be documented and additional pairs of BP readings will be taken as necessary to complete 3 valid pairs of Reference/Test BP comparisons. A maximum total of 8 pairs of Reference/Test BP readings can be taken ( 5 additional ones) until 3 valid pairs are obtained. Examples of observer disagreement and repeat pairs of measurements required are shown in Figure 1. The agreement between the observers during the validation study must be reported (mean difference, $S D$ and range, number of measurements with disagreement).

- Reference BP variability: Subjects with $\mathrm{SBP}$ difference $>12 \mathrm{mmHg}$ and/or DBP $>8 \mathrm{mmHg}$ in any 2 of the 4 Reference (average of 2 observers) BP measurements $R 1$ to $R 4$ must be excluded. Measurement $R O$ will not be used in the evaluation of reference BP variability. If a participant's reference BP measurements do not fulfill this requirement, then 2 consecutive valid pairs of reference measurements (instead of the 3 required) that fulfill this requirement can be included in the analysis, but in a maximum of $10 \%$ of the participants.

\section{Test device BP measurement}

- The test device and cuffs must be used according to the manufacturer's instructions following a period of familiarization of the supervisor with the device function. Any issues in test BP measurements must be reported.

\section{Validation studies in general and special populations}

- General population studies: Requirements are presented in Table 3.

- Special populations studies: These are defined as subjects for whom there is theoretical and clinical evidence of different accuracy of BP monitors to that in the general population. At the present these include: (i) age $<3$ years; (ii) pregnancy including preeclampsia (see below); (iii) arm circumference $>42 \mathrm{~cm}$; (iv) atrial fibrillation (there is no agreed reference BP measurement and validation procedure for patients with atrial fibrillation). Subjects aged $12-18$ or $>80$ years, patients with end-stage renal disease or peripheral artery disease, or diabetes with organ damage are possible special groups, but there is uncertainty on the adequacy of existing data suggesting altered accuracy of BP monitors and more research is needed. Requirements for validation studies in special populations are presented in Table 4.

- Children (age 3-12 years): Requirements are presented in Table 5. 
- $\quad$ Pregnancy (including preeclampsia): Requirements are presented in Table 6.

\section{Validation data analysis - Pass/fail criteria}

- The validation data analysis is performed using the Reference BP measurements $R 1-R 4$ and the Test device measurements T1-T3. Each of the Reference BP measurements is the average of the simultaneous readings of the two observers. Measurements $R O$ and $T O$ are not used in the evaluation of the test device accuracy.

- Each of the Test device measurements is compared against the average of the previous and succeeding Reference BP readings (e.g. $T 1$ versus the average of $R 1-R 2, T 2$ versus average of $R 2$ $R 3, T 3$ versus average of $R 3-R 4)$. Differences are calculated by subtracting the Reference $(R) \mathrm{BP}$ measurement from the Test $(T)$ device measurement. Thus, in an 85-subject study there are 255 paired BP comparisons.

- Standardized Bland-Altman scatterplots of the Test-Reference BP differences against their average value, which give a visual presentation of the raw data, must be provided as in Figure 1 of the full validation study paper example presented in the end of this article ( $x$-axis in SBP range $80-190 \mathrm{mmHg}$ and DBP $30-140 \mathrm{mmHg}$; $y$-axes with errors from -30 to $+30 \mathrm{mmHg}$ for SBP and DBP plots); horizontal reference lines at $5 \mathrm{mmHg}$ intervals from +15 to $-15 \mathrm{mmHg}$; vertical lines to represent BP distribution boundaries; BP differences $>30 \mathrm{mmHg}$ plotted at point 30 $\mathrm{mmHg}$ and $<-30 \mathrm{mmHg}$ at $-30 \mathrm{mmHg}[3,6]$.

- For a device to pass the AAMI/ESH/ISO Universal Standard (ISO 81060-2:2018) two criteria must be fulfilled:

a. Criterion 1 (for individual BP readings): The mean BP difference (Test minus Reference BP for all 255 pairs of measurements) must be $\leq 5 \mathrm{mmHg}$ and its SD $\leq 8 \mathrm{mmHg}$ for SBP and DBP (classic AAMI criterion [3-5,10]).

b. Criterion 2 (for individual subjects): the SD of 85 averaged BP differences (Test minus Reference BP per subject) must be within a threshold defined by the mean Test-Reference BP difference listed in Table 7 for SBP and DBP [3-5]. The Criterion 2 is applied only in 85subject study samples.

\section{Validation study data reports}

For the optimal execution and data reporting of validation studies according to the AAMI/ESH/ISO Universal Standard (ISO 81060-2:2018) the following tools are provided:

a. Forms for the supervisor and observers of validation studies for compiling individual subjects' data while performing a study (Figure 2).

b. Checklist for investigators performing validation studies and for reviewers of validation study papers submitted for publication in scientific journals or other peer review organizations (Table 8).

c. Example of full paper reporting a validation study with complete results, tables and figure (see end of this article).

The above tools aim to prevent protocol violations in performing validation studies and incomplete data reporting (missing or unclear data), which are particularly common and often missed by the peer-review process of scientific journals [7,11-13].

\section{Acknowledgments}

We thank validation team members in Athens, London, Oxford, Padova and Paris for helping in the development of practical tools for performing and reporting a validation study. 


\section{References}

1. O'Brien E, Dolan E, Stergiou GS. Achieving reliable blood pressure measurements in clinical practice: It's time to meet the challenge. J Clin Hypertens 2018;20:1084-1088.

2. Stergiou GS, Alpert BS, Mieke S, Wang J, O'Brien E. Validation protocols for blood pressure measuring devices in the 21st century. J Clin Hypertens 2018;20:1096-1099.

3. Stergiou GS, Alpert B, Mieke S, Asmar R, Atkins N, Eckert S, Frick G, Friedman B, GraßI T, Ichikawa T, loannidis JP, Lacy P, McManus R, Murray A, Myers M, Palatini P, Parati G, Quinn D, Sarkis J, Shennan A, Usuda T, Wang J, Wu CO, O'Brien E. A universal standard for the validation of blood pressure measuring devices: Association for the Advancement of Medical Instrumentation/European Society of Hypertension/International Organization for Standardization (AAMI/ESH/ISO) Collaboration Statement. Hypertension 2018;71:368-374 and J Hypertens 2018;36:472-478.

4. International Organization for Standardization. ISO 81060-2:2018. Non-invasive sphygmomanometers Part 2: Clinical investigation of intermittent automated measurement type. https://www.iso.org/standard/73339.html. Accessed 7 Dec. 2018.

5. Association for the Advancement of Medical Instrumentation (AAMI). ANSI/AAMI/ISO 81060-2:2018. Non-invasive sphygmomanometers - Part 2: Clinical investigation of intermittent automated measurement type. http://my.aami.org/store. In press.

6. O'Brien E, Atkins N, Stergiou G, Karpettas N, Parati G, Asmar R, Imai Y, Wang J, Mengden T, Shennan A; Working Group on Blood Pressure Monitoring of the European Society of Hypertension. European Society of Hypertension International Protocol revision 2010 for the validation of blood pressure measuring devices in adults. Blood Press Monit. 2010;15:23-38.

7. Stergiou GS, Karpettas N, Atkins N, O’Brien E. European Society of Hypertension International Protocol for the validation of blood pressure monitors: a critical review of its application and rationale for revision. Blood Press Monit. 2010;15:39-48.

8. Stergiou GS, Asmar R, Myers M, Palatini P, Parati G, Shennan A, Wang J, O'Brien E; European Society of Hypertension Working Group on Blood Pressure Monitoring and Cardiovascular Variability. Improving the accuracy of blood pressure measurement: the influence of the European Society of Hypertension International Protocol (ESH-IP) for the validation of blood pressure measuring devices and future perspectives. J Hypertens. 2018;36:479-487.

9. O'Brien E, Petrie J, Littler WA, de Swiet M, Padfield PL, Altman D, et al. The British Hypertension Society Protocol for the evaluation of blood pressure measuring devices. J Hypertens 1993; 11(Suppl 2):S43-S62.

10. Association for the Advancement of Medical Instrumentation. The National Standard of Electronic or Automated Sphygmomanometers. Arlington, VA: AAMI; 1987.

11. Hodgkinson JA, Sheppard JP, Heneghan C, Martin U, Mant J, Roberts N, McManus RJ. Accuracy of ambulatory blood pressure monitors: a systematic review of validation studies. J Hypertens. 2013;31:239250.

12. Boubouchairopoulou N, Kollias A, Atkins N, O'Brien E, Stergiou GS. Validation of blood pressure monitors using the AAMI and ISO protocols: An overview of their recent application. European Society of Hypertension 26th Meeting (Abstract). J Hypertens. 2016;34:e286.

13. McManus R, Lacy P, Clark C, Chapman N, Lewis P; British and Irish Hypertension Society Blood Pressure Measurement Working Party. Reporting of blood pressure monitor validation studies. Blood Press Monit. 2018;23:214-215 
Table 1.

Standard validation procedure for reference and test device BP measurements in same arm sequential method (with permission from [3]).

\section{Initial BP measurements*}

1. Take Reference BP measurement by the 2 observers $\quad R O$

2. Take Test device BP measurement TO

\section{Validation BP measurements for accuracy evaluation}

3. Take 1st Reference BP measurement by the 2 observers $\quad R 1$

4. Take 1st Test device BP measurement $\quad T 1$

5. Take 2nd Reference BP measurement by the 2 observers R2

6. Take 2nd Test device BP measurement $\quad$ T2

7. Take 3rd Reference BP measurement by the 2 observers R3

8. Take 3rd Test device BP measurement $\quad$ T3

9. Take 4th Reference BP measurement by the 2 observers $R 4$

$\mathrm{BP}$, blood pressure; * Measurement $R O$ shall not be used in the evaluation of Reference BP distribution and variability criteria. Measurements $R O$ and $T O$ shall not be used in the evaluation of the test device accuracy.

Table 2.

Example of cuff use (inflatable bladder dimensions) for reference auscultatory blood pressure measurement in a general population validation study including children (with permission from [3]).

\begin{tabular}{lcccccc} 
Bladder & \multicolumn{6}{c}{ Participant's mid-arm circumference $(\mathrm{cm})$} \\
dimensions & $12-15$ & $15-18$ & $18-23$ & $23-28$ & $28-35$ & $33-42$ \\
\hline Length $(\mathrm{cm})$ & 12 & 15 & 18 & 23 & 28 & 33 \\
Width $(\mathrm{cm})$ & 6 & 7 & 9 & 12 & 14 & 16 \\
\hline
\end{tabular}


Table 3.

Requirements for a validation study in general population.

\begin{tabular}{|c|c|}
\hline Sample size & $\begin{array}{l}\text { At least } 85 \text { consecutive subjects fulfilling the standard requirements } \\
\text { providing at least } 255 \text { paired (test and reference) BP measurements. }\end{array}$ \\
\hline Age & $>12$ years \\
\hline Gender & $\geq 30 \%$ men and $\geq 30 \%$ women \\
\hline Treatment & Untreated or treated \\
\hline Exclusion criteria & $\begin{array}{l}\text { Special populations (see below), presence of any arrhythmia and any } \\
\text { issues during the validation procedure. Excluded subjects must be } \\
\text { reported with reasons for exclusion. }\end{array}$ \\
\hline \multirow{3}{*}{$\begin{array}{l}\text { Reference BP } \\
\text { distribution criteria } \\
\text { (readings } R 1-R 4 \text { ) }\end{array}$} & $\begin{array}{l}\text { - Of the SBP readings } \geq 5 \% \text { must be } \leq 100 \mathrm{mmHg}, \geq 5 \% \text { must be } \geq 160 \\
\mathrm{mmHg} \text { and } \geq 20 \% \geq 140 \mathrm{mmHg} \text {. }\end{array}$ \\
\hline & $\begin{array}{l}\text { - Of the DBP readings } \geq 5 \% \text { must be } \leq 60 \mathrm{mmHg} \text {, } \geq 5 \% \text { must be } \geq 100 \\
\mathrm{mmHg} \text { and } \geq 20 \% \geq 85 \mathrm{mmHg} \text {. }\end{array}$ \\
\hline & $\begin{array}{l}\text { - Measurement } R O \text { shall not be used in the evaluation of reference BP } \\
\text { distribution criteria. }\end{array}$ \\
\hline \multirow[t]{3}{*}{$\begin{array}{l}\text { Cuff sizes stratified } \\
\text { subgroups }\end{array}$} & $\begin{array}{l}\text { For test devices with a single cuff } \geq 40 \% \text { of the subjects must have } \\
\text { arm circumference within the upper half of the specified range of } \\
\text { use of the cuff, } \geq 40 \% \text { within the lower half, } \geq 20 \% \text { within the higher } \\
\text { quarter, } \geq 20 \% \text { within the lower quarter, } \geq 10 \% \text { within the higher octal } \\
\text { and } \geq 10 \% \text { within the lower octal of the specified range of use of the } \\
\text { cuff. }\end{array}$ \\
\hline & $\begin{array}{l}\text { For test devices that have multiple ( } n \text { ) cuffs, each cuff must be tested } \\
\text { on at least } 1 /(2 \times n) \text { of the subjects (e.g. in a } 85 \text {-subject study, for a } \\
\text { device with } 2 \text { cuffs } \geq 21 \text { subjects per cuff and for } 3 \text { cuffs } \geq 14 \text { subjects } \\
\text { per cuff). For each test device, } \geq 40 \% \text { of the subjects included using } \\
\text { this cuff must have arm circumference within the upper half of the } \\
\text { specified range of use of the cuff and } \geq 40 \% \text { within the lower half. }\end{array}$ \\
\hline & $\begin{array}{l}\text { The mean test-reference BP difference and SD per cuff subgroup } \\
\text { must be reported (these are not pass/fail criteria for the test device). }\end{array}$ \\
\hline
\end{tabular}

BP, blood pressure; SBP, systolic BP; DBP, diastolic BP; SD, standard deviation. 
Table 4.

Requirements for validation studies in special populations.

\begin{tabular}{ll}
\hline Sample & - At least 35 subjects to be investigated only after a full 85 -subject general \\
size & population study has been successfully completed. \\
& - At least 85 subjects for devices intended only for use in special population. \\
Selection & - For children aged 3-12 years see table 5 and for pregnant women table 6. \\
Analysis & - Special population study data must be analyzed and reported independently \\
& of the general population study data. \\
& - For 85 -subject special population studies both validation Criteria 1 and 2 are \\
& used. For 35 -subject studies (performed after a successful 85 -subject general \\
& population study) only validation Criterion 1 is used.
\end{tabular}

Table 5.

Requirements for a validation study in children (age 3-12 years).
Sample size - Devices intended for children and older subjects: 35 children aged 3-12 years can be included together with 50 subjects $>12$ years in an 85 -subject study (gender and BP distribution criteria to apply in the total sample).
- Devices intended only for children: $\geq 85$ children aged 3-12 years are required.
- Devices with a special BP measurement mode for children: $\geq 35$ children aged 3- 12 years are required (after a successful 85 -subject study in general population; BP distribution requirements do not apply).
Reference BP - Korotkoff K1 and K5 shall be used. If K1 or K5 are not audible, the child is excluded.
Analysis $\quad$ For 85-child studies both the validation Criteria 1 and 2 are used.
- For 85 -subject studies including 35 children and 50 older subjects, both the validation criteria 1 and 2 apply in the total 85-subject sample. Criterion 1 (Test minus Reference BP difference) must also be reported separately for children (not a pass/fail criterion).
- For a 35-child study of a device with a special BP measurement mode for children (performed after a successful 85-subject general population study) only validation Criterion 1 is used.


Table 6.

Requirements for a validation study in pregnancy including preeclampsia.

\begin{tabular}{|c|c|}
\hline Sample size & $\begin{array}{l}\text { - } 45 \text { pregnant women after a successful } 85 \text {-subject study in general population } \\
\text { (otherwise } 85 \text { pregnant women needed). }\end{array}$ \\
\hline $\begin{array}{l}\text { Selection } \\
\text { criteria }\end{array}$ & $\begin{array}{l}\text { 15 } \pm 1 \text { normotensive women in second or third trimester of pregnancy (BP } \\
<140 / 90 \mathrm{mmHg} \text { ), } 15 \pm 1 \text { with hypertension in second or third trimester of } \\
\text { pregnancy ( } \mathrm{SBP} \geq 140 \mathrm{mmHg} \text { and/or } \mathrm{DBP} \geq 90 \mathrm{mmHg} \text { without proteinuria), and } \\
15 \pm 1 \text { women with preeclampsia (SBP } \geq 140 \mathrm{mmHg} \text { and/or DBP } \geq 90 \mathrm{mmHg} \text { with } \\
\text { proteinuria). } \\
\text { - Arm/cuff and BP distribution criteria will not be applied. }\end{array}$ \\
\hline $\begin{array}{l}\text { Reference BP } \\
\text { Analysis }\end{array}$ & $\begin{array}{l}\text { - Korotkoff } \mathrm{K} 1 \text { and } \mathrm{K} 5 \text { shall be used. If } \mathrm{K} 1 \text { or } \mathrm{K} 5 \text { are not audible, the woman is } \\
\text { excluded. } \\
\text { For } 85 \text {-woman studies both the validation Criteria } 1 \text { and } 2 \text { are used. } \\
\text { - For } 45 \text {-woman studies (performed after a successful } 85 \text {-subject general } \\
\text { population study) only validation Criterion } 1 \text { is applied in the total sample. } \\
\text { - Data from preeclamptics (mean BP difference and SD) must be reported } \\
\text { separately (this is not a pass/fail criterion). }\end{array}$ \\
\hline
\end{tabular}

Table 7.

Validation Criterion 2: Maximum permissible standard deviation for the 85 averaged BP differences (test versus reference BP per subject) within a threshold defined by the mean BP difference (with permission from [4]).

\begin{tabular}{|c|c|c|c|c|c|c|c|c|c|c|}
\hline \multirow{2}{*}{$\begin{array}{r}\text { Mean BP } \\
\text { difference }\end{array}$} & \multicolumn{10}{|c|}{$\begin{array}{l}\text { Maximum permissible standard deviation as function of the mean BP difference } \\
\qquad(\mathrm{mmHg})\end{array}$} \\
\hline & 0.0 & 0.1 & 0.2 & 0.3 & 0.4 & 0.5 & 0.6 & 0.7 & 0.8 & 0.9 \\
\hline \pm 0 & 6.95 & 6.95 & 6.95 & 6.95 & 6.93 & 6.92 & 6.91 & 6.90 & 6.89 & 6.88 \\
\hline \pm 1 & 6.87 & 6.86 & 6.84 & 6.82 & 6.80 & 6.78 & 6.76 & 6.73 & 6.71 & 6.68 \\
\hline \pm 2 & 6.65 & 6.62 & 6.58 & 6.55 & 6.51 & 6.47 & 6.43 & 6.39 & 6.34 & 6.30 \\
\hline \pm 3 & 6.25 & 6.20 & 6.14 & 6.09 & 6.03 & 5.97 & 5.89 & 5.83 & 5.77 & 5.70 \\
\hline \pm 4 & 5.64 & 5.56 & 5.49 & 5.41 & 5.33 & 5.25 & 5.16 & 5.08 & 5.01 & 4.90 \\
\hline \pm 5 & 4.79 & - & - & - & - & - & - & - & - & - \\
\hline
\end{tabular}

Example: For mean BP difference $\pm 4.2 \mathrm{mmHg}$ the maximum permissible standard deviation is $5.49 \mathrm{mmHg}$ (gray boxes). BP, blood pressure. 
TABLE 8. CHECKLIST FOR INVESTIGATORS AND REVIEWERS OF VALIDATION STUDIES OF BLOOD PRESSURE MEASURING DEVICES PERFORMED USING THE AAMI/ESH/ISO UNIVERSAL STANDARD (ISO 81060-2:2018)

European Society of Hypertension Working Group on Blood Pressure Monitoring and Cardiovascular Variability

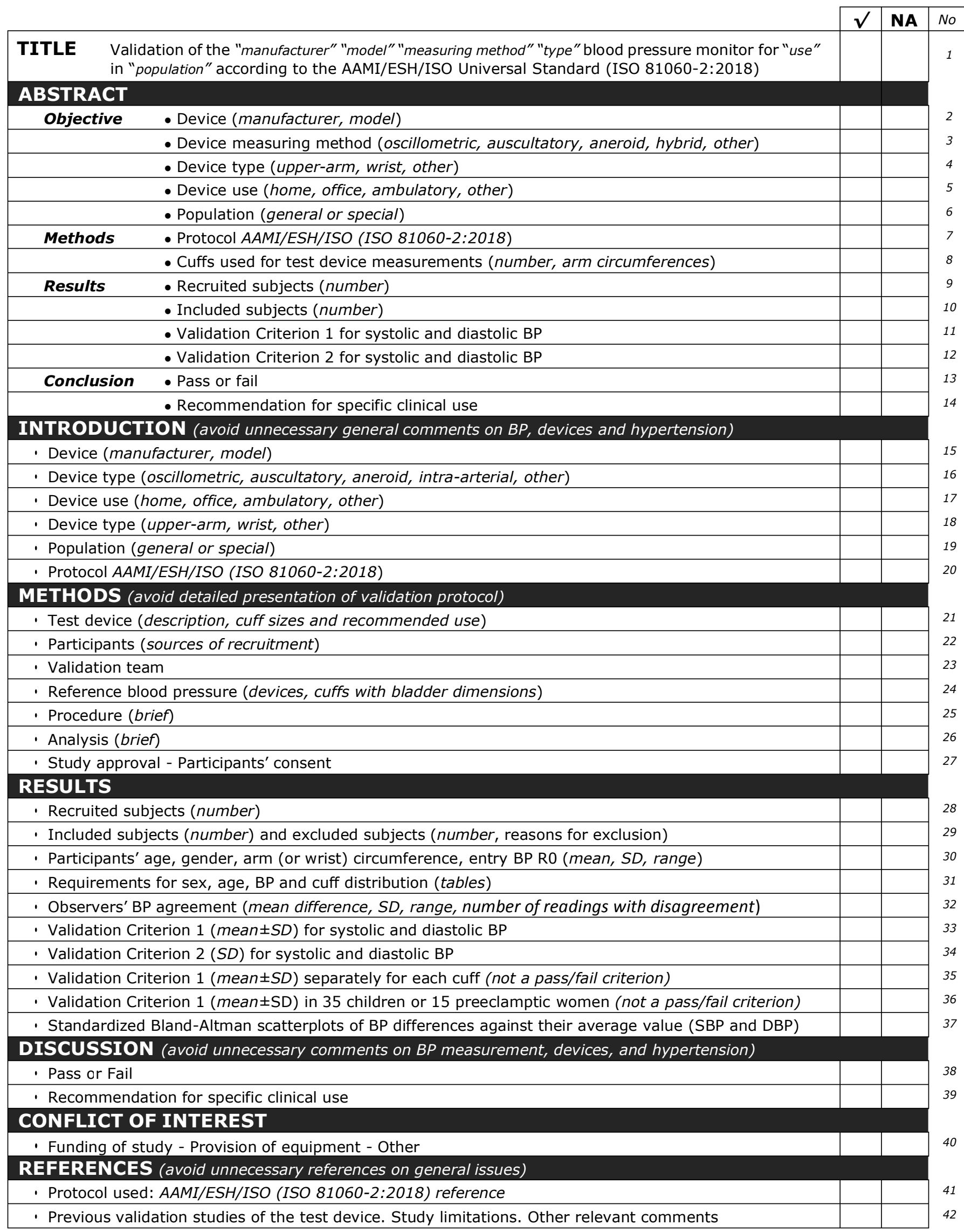




\section{Legends to figures}

\section{Figure 1.}

Examples of inter-observer reference blood pressure measurements disagreement (black boxes) and repeat pairs (valid consequent reference-test-reference) of measurements required (dotted circles represent reference and test measurements to be discarded; max 8 pairs are allowed not including RO and TO).

\section{Figure 2.}

Forms for supervisor and observers of validation studies to fill individual subjects' data while performing a study. 


\title{
Example of full paper reporting an 85-subject general population validation study of a blood pressure monitor according to the AAMI/ESH/ISO Universal Standard (ISO 81060-2:2018)
}

Title: Validation of the NovelBP-99HT oscillometric blood pressure monitor for professional office use in general population according to the AAMI/ESH/ISO Universal Standard.

\begin{abstract}
Objective: To evaluate the accuracy of the NovelBP-99HT oscillometric upper-arm professional office blood pressure (BP) monitor in general population according to the Association for the Advancement of Medical Instrumentation/European Society of Hypertension/International Organization for Standardization (AAMI/ESH/ISO) Universal Standard (ISO 81060-2:2018).

Methods: Subjects were recruited to fulfil the age, gender, BP and cuff distribution criteria of the AAMI/ESH/ISO Universal Standard in general population using the same arm sequential BP measurement method. Two cuffs of the test device were used for arm circumference 22-32 (medium) and 32-42 cm (large).

Results: Ninety-one subjects were recruited and 85 were analyzed. For validation Criterion 1 , the mean $\pm S D$ of the differences between the test device and reference BP readings was -

2.3 $\pm 6.2 / 0.6 \pm 4.8 \mathrm{mmHg}$ (systolic/diastolic). For validation Criterion 2, the SD of the averaged BP differences between the test device and reference BP per subject was $4.62 / 4.01 \mathrm{mmHg}$ (systolic/diastolic).

Conclusions: The NovelBP-99HT professional office BP monitor fulfilled the requirements of the AAMI/ESH/ISO Universal Standard (ISO 81060-2:2018) in general population and can be recommended for clinical use.
\end{abstract}

Keywords: accuracy, blood pressure measurement, validation, automated, oscillometric, office.

Conflict of interest: None for all authors.

Funding: The study was funded by NovelBP, London, UK.

\section{INTRODUCTION}

This study assessed the blood pressure (BP) measurement accuracy of the professional oscillometric upper-arm cuff device NovelBP-99HT (NovelBP, London, UK) developed for office BP measurement using the Association for the Advancement of Medical Instrumentation/European Society of Hypertension/International Organization for Standardization (AAMI/ESH/ISO) Universal Standard (ISO 81060-2:2018) in general population [1].

\section{METHODS}

Test device

The NovelBP-99HT is an automated oscillometric upper-arm BP monitor developed for professional use in the office. The device has a medium size cuff for arm circumference $22-32 \mathrm{~cm}$ and a large cuff for arm 32-42 cm, which were used in the validation study according to the manufacturers' instructions. Two identical devices were provided by the manufacturer for the validation study together with a written declaration that they were standard production models. One of them was randomly selected for the validation procedure. 


\section{Participants}

According to the AAMI/ESH/ISO Universal Standard, for a general population validation study of a BP monitor at least 85 subjects aged $>12$ years are required [1]. Subjects were recruited from patients attending the outpatient hypertension clinic and from hospital staff.

\section{Validation team}

The study was conducted by a supervisor and 2 trained observers who were experienced in BP measurement research and were standardized for their agreement in BP measurement before the study initiation [1].

\section{Reference BP}

Two connected (Y-tube) standard mercury sphygmomanometers (Supermeter, Meditools, Chicago, USA), which had been calibrated before the study initiation were used for simultaneous reference auscultatory BP measurements by two observers using a dual-head teaching stethoscope (Doctor Educ-3, Cardiocare, Berlin, Germany). Three cuffs with inflatable bladder dimensions 12X23, 14X28, $16 \times 33 \mathrm{~cm}$ respectively were used so that the length would be at $75-100 \%$ of the individual participant's midarm circumference and the width at $37-50 \%$ [1].

\section{Procedure}

The same arm sequential method was applied [1], which includes 2 entry BP measurements (reference R0 and test device T0) followed by 4 reference measurements (R1, R2, R3, R4) taken alternately with 3 test device measurements $(T 1, T 2, T 3)$. All measurements were taken on the left arm. The observers were blinded to each other's readings and the test device results. The supervisor recorded the test device measurements and checked the observers' measurements. In case of disagreement between the observers, additional pairs of measurements were performed. A maximum of 8 pairs of BP determinations was allowed after which the subject was excluded.

\section{Analysis}

The AAMI/ESH/ISO Universal Standard (ISO 81060-2:2018) requirements were strictly followed [1].

\section{Study approval}

The study protocol was approved by the hospital scientific committee. All participants signed informed consent for study participation.

\section{RESULTS}

Ninety-one individuals were recruited and 85 were analyzed. Reasons for exclusion are presented in Table 1. The participants' characteristics are presented in Table 2. The requirements for age, gender, BP and cuff distribution were fulfilled [1]. The mean BP difference between the simultaneous observers' measurements was $0.3 \pm 1.6[\mathrm{SD}] /-0.2 \pm 1.7 \mathrm{mmHg}$ (systolic/diastolic, range -4 to $4 \mathrm{mmHg}$ ). There were $18 \mathrm{BP}$ readings with inter-observer disagreement $>4 \mathrm{mmHg}$.

The distribution of the reference BP measurements R1-R4 is presented in Table 3. The medium cuff of the test device was used in 43 subjects and the large in 42. Participants with arm circumference within the upper/lower half of the specified range of use of the medium cuff were 24/19 respectively (56/44\%) and of the large cuff $17 / 25$ (40/60\%). The validation analysis is shown in Table 4. Both the validation Criteria 1 and 2 suggested 'pass' for systolic and diastolic BP. Validation Criterion 1 results per cuff size are presented in Table 5. Standardized Bland-Altman scatterplots of the test-reference BP differences against their average are shown in Figure 1. 
Table 1. Participants recruited and excluded from the analysis.

\begin{tabular}{lc}
\hline & Subjects \\
\hline Recruited & 91 \\
Excluded & 6 \\
Reasons for exclusion & \\
- Reference BP variability & 4 \\
(>12/8 mmHg for systolic/diastolic) & \\
- K sounds not audible & 1 \\
- Talking during BP measurements & 1 \\
\hline Analyzed & 85 \\
\hline
\end{tabular}

Table 2. Participants' characteristics $(n=85)$.

\begin{tabular}{lcc}
\hline & Mean \pm SD & Range \\
\hline Age (years) & $54.8 \pm 12.1$ & $22-75$ \\
Gender (male/female) & $45 / 40$ & - \\
Arm circumference $(\mathrm{cm})$ & $32.1 \pm 4.1$ & $23.0-39.8$ \\
Entry SBP RO $(\mathrm{mmHg})$ & $129.7 \pm 18$ & $82-174$ \\
Entry DBP RO $(\mathrm{mmHg})$ & $79.8 \pm 11.7$ & $48-112$ \\
\hline
\end{tabular}

Table 3. Distribution of reference BP measurements (R1-R4).

\begin{tabular}{cccc}
\hline SBP & $\leq 100 \mathrm{mmHg}$ & $\geq 160 \mathrm{mmHg}$ & $\geq 140 \mathrm{mmHg}$ \\
& $9.8 \%$ & $6.7 \%$ & $28.2 \%$ \\
\hline DBP & $\leq 60 \mathrm{mmHg}$ & $\geq 100 \mathrm{mmHg}$ & $\geq 85 \mathrm{mmHg}$ \\
& $5.1 \%$ & $5.9 \%$ & $34.9 \%$ \\
\hline
\end{tabular}

Table 4. Validation study results.

\begin{tabular}{lccc}
\hline & Pass & \multicolumn{2}{c}{ Achieved } \\
& requirement & SBP & DBP \\
\hline Criterion 1 (255 BP pairs) & & & \\
Mean BP difference (mmHg) & $\leq 5$ & -2.3 & 0.6 \\
SD (mmHg) & $\leq 8$ & 6.2 & 4.8 \\
& & Pass & Pass \\
\hline Criterion 2 (85 Subjects) & $\leq 6.55 / 6.91$ & 4.62 & 4.01 \\
SD (mmHg, SBP/DBP) & & Pass & \multicolumn{2}{c}{ Pass } \\
& & \multicolumn{3}{c}{ Pass } \\
\hline
\end{tabular}


Table 5. Test device cuff size distribution and results (criterion 1).

\begin{tabular}{lccc}
\hline $\begin{array}{l}\text { Test device } \\
\text { cuff size }\end{array}$ & $\begin{array}{c}\text { Participants } \\
(\%)\end{array}$ & $\begin{array}{c}\text { Mean SBP difference } \\
\pm S D(\mathbf{m m H g})\end{array}$ & $\begin{array}{c}\text { Mean DBP difference } \\
\pm \text { SD }(\mathbf{m m H g})\end{array}$ \\
\hline Medium & $43(51)$ & $-2.2 \pm 6.0$ & $1.6 \pm 4.6$ \\
Large & $42(49)$ & $-2.3 \pm 6.4$ & $-0.4 \pm 4.9$ \\
\hline
\end{tabular}

Figure 1. Standardized Bland-Altman scatterplots of the Test-Reference BP differences against their average [1].

\section{DISCUSSION}

This study shows that NovelBP-99HT office BP monitor fulfils the accuracy criteria of the AAMI/ESH/ISO Universal Standard (ISO 81060-2:2018) in a general population and can be recommended for clinical use.

\section{REFERENCES}

1. Stergiou G, Palatini P, Asmar R, loannidis JP, Kollias A, Lacy P, McManus R, Myers M, Parati G, Shennan A, Wang J, $O$ 'Brien $E$, on behalf of the European Society of Hypertension Working Group on Blood Pressure Monitoring. Recommendations and Practical Guidance for Performing and Reporting Validation Studies according to the Universal Standard for the Validation of Blood Pressure Measuring Devices by the Association for the Advancement of Medical Instrumentation/European Society of Hypertension/International Organization for Standardization (AAMI/ESH/ISO). J Hypertens 2019;37:XXX-XXX. 